\title{
MULTI-TARGET TRACKING OF PACKED YEAST CELLS
}

\author{
Ricard Delgado-Gonzalo*, Nicolas Dénervaud ${ }^{\dagger}$,Sebastian Maerkl ${ }^{\dagger}$, and Michael Unser* \\ *Biomedical Imaging Group and ${ }^{\dagger}$ Laboratory of Biological Network Characterization \\ École Polytechnique Fédérale de Lausanne (EPFL), Switzerland
}

\begin{abstract}
The tracking of cell populations in time-lapse microscopy images enables high-throughput spatiotemporal measurements of cell dynamics. In this paper, we present a new algorithm to simultaneously track many cells in crowded areas. The algorithm runs in real time and deals with thousands of cells. The main contribution of this paper is that the algorithm is able to maintain the spatiotemporal consistency of the tracks in crowded areas, even when the temporal resolution is coarse. We validate our approach in terms of its ability to track yeast cells.
\end{abstract}

Index Terms - time-lapse microscopy, tracking, regularization, crowd, graph matching, yeast, interaction networks.

\section{INTRODUCTION}

Due to the dynamic nature of biological systems, it is highly desirable to quantify their evolution through time to improve our understanding of their interaction networks. Large-scale time-lapse imaging of cells is nowadays performed routinely thanks to the automatization achieved in the field of light microscopy. The obtained datasets are such that it is not possible to analyze them manually within any tolerable amount of time [1].

Present-day efforts in cell tracking are application-oriented and depend on different methodological approaches. Several strategies exist to perform inter-frame target association. The most straightforward is to link each detected target with the closest one in the subsequent frame, where the used distance may include similarity measures [2]. This simplicity is lost when the cells are densely packed and interact with each other. Joint optimization tracing techniques provide good results in such cases [3, 4].

The usual probabilistic approach to cell tracking is the Bayesian filtering framework and involves statistical dynamical models [5, 6, 7]. However, incorporating a dynamical model is hazardous since the behavior of biological systems may vary under different environments. It has also been proposed to use graph-theoretic approaches. In this framework the solution is obtained using standard graph optimization algorithms on a weighted graph composed of the detected targets and all possible correspondences [8,9].

In this paper, we present a new and fast algorithm which is specialized for tracking biological cells within crowds. It relies on graph-theoretic techniques to minimize a cost functional that models the characteristic motion in highly packed scenes and imposes a certain level of coherence in the displacement field while being capable of handling large movements (i.e., coarse temporal resolution).

This work was supported by the Swiss Initiative in Systems Biology (SystemsX).

\section{PROBABILISTIC FORMULATION}

In this section, we develop a probabilistic formulation inspired by the Bayesian filtering framework. Our setting is ruled by probabilistic graphs and leads to a simple implementation.

\subsection{Notation}

We denote $\mathrm{M}^{k}=\left\{\boldsymbol{m}_{j}^{k}\right\}_{j=1 \ldots N_{k}}$ the set of measurements $\boldsymbol{m}_{j}^{k}=$ $\left(\boldsymbol{x}_{j}^{k}, \boldsymbol{s}_{j}^{k}\right)$ at frame $k$, where each measurement contains information about the position $\boldsymbol{x}_{j}^{k}$ and features $\boldsymbol{s}_{j}^{k}$ of each target. Analogously, we define the set of positions and features at a given frame by $\mathrm{X}^{k}=$ $\left\{\boldsymbol{x}_{j}^{k}\right\}_{j=1 \ldots N_{k}}$ and $\mathrm{S}^{k}=\left\{\boldsymbol{s}_{j}^{k}\right\}_{j=1 \ldots N_{k}}$.

We are interested in finding the best association between two consecutive frames given the knowledge of the measurements in these frames. We define a pairing as a subset $\mathrm{A}^{k} \subset \Omega=$ $\left\{1 \ldots N_{k-1}\right\} \times\left\{1 \ldots N_{k}\right\}$, where $(i, j) \in \mathrm{A}^{k}$ indicates that the measurement $\boldsymbol{m}_{i}^{k-1}$ in frame $(k-1)$ corresponds to the measurement $\boldsymbol{m}_{j}^{k}$ in frame $k$. We restrict the admissible solution by disallowing multiple assignments to the same measurement. Formally, given $(i, j),(l, n) \in \mathrm{A}^{k}$, then $\boldsymbol{m}_{i}^{k-1}=\boldsymbol{m}_{l}^{k-1}$ if and only if $\boldsymbol{m}_{j}^{k}=\boldsymbol{m}_{n}^{k}$ (see Fig.1). Note that the cell-tracking problem is equivalent to finding the corresponding $\mathrm{A}^{k}$ at each transition interval.

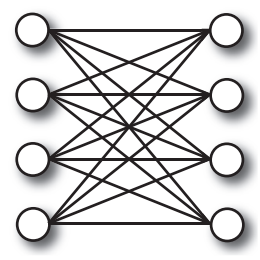

(a)

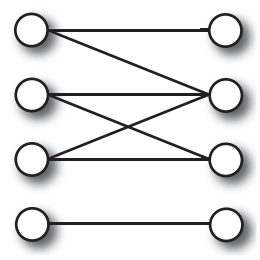

(b)

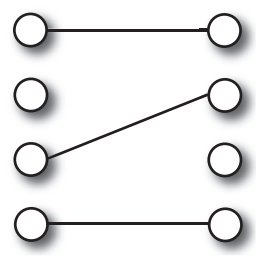

(c)
Fig. 1. Graph representation of the sets $\mathrm{M}^{k-1}$ (left side), $\mathrm{M}^{k}$ (right side) with (a) all possible correspondences given by $\Omega$, (b) the most likely correspondences given by $G$, and (c) a possible pairing $\mathrm{A}^{k}$.

\subsection{Bayesian Formulation}

We consider the measurements sets as random variables. This implies that we are also looking for a pairing which is a random element within the space of all possible pairings. We are interested in finding a pairing that maximizes its conditional probability given the known information, that is, the measurements

$$
\tilde{\mathrm{A}}^{k}=\underset{\mathrm{A}^{k} \subset \Omega}{\arg \max }\left\{P\left(\mathrm{~A}^{k} \mid \mathrm{M}^{k-1}, \mathrm{M}^{k}\right)\right\} .
$$


Using an approach similar to the one in the Bayesian filtering framework, and assuming mutual independence between the positions and the features, we restate the maximization criteria of (1) as

$$
\tilde{\mathrm{A}}^{k}=\underset{\mathrm{A}^{k} \subset \Omega}{\arg \max }\left\{P\left(\mathrm{~A}^{k}\right) P\left(\mathrm{X}^{k} \mid \mathrm{X}^{k-1}, \mathrm{~A}^{k}\right) P\left(\mathrm{~S}^{k} \mid \mathrm{S}^{k-1}, \mathrm{~A}^{k}\right)\right\},
$$

where $P\left(\mathrm{~A}^{k}\right)$ reflects our prior knowledge about the preferred pairings, $P\left(\mathrm{X}^{k} \mid \mathrm{X}^{k-1}, \mathrm{~A}^{k}\right)$ corresponds to a measure of likelihood of the positions given that we know the position in the previous frame through the pairing $\mathrm{A}^{k}$, and $P\left(\mathrm{~S}^{k} \mid \mathrm{S}^{k-1}, \mathrm{~A}^{k}\right)$ corresponds to a measure of how likely the feature values are given the knowledge of the feature values in the previous frame through the pairing $\mathrm{A}^{k}$.

We finally model those probabilities as

$$
\begin{aligned}
P\left(\mathrm{X}^{k} \mid \mathrm{X}^{k-1}, \mathrm{~A}^{k}\right) & \propto \mathrm{e}^{-\sum_{v \in \mathrm{A}^{k}} \mu_{X}\left(v, \mathrm{X}^{k-1}, \mathrm{X}^{k}, \mathrm{~A}^{k}\right)} \\
P\left(\mathrm{~S}^{k} \mid \mathrm{S}^{k-1}, \mathrm{~A}^{k}\right) & \propto \mathrm{e}^{-\sum_{v \in \mathrm{A}^{k}} \mu_{S}\left(v, \mathrm{~S}^{k-1}, \mathrm{~S}^{k}, \mathrm{~A}^{k}\right)},
\end{aligned}
$$

where $\mu_{X}$ is a compatibility measure of the joint movement and $\mu_{S}$ is a feature compatibility measure.

\section{EFFICIENT GRAPH-BASED ALGORITHM}

We now cast the problem of finding the best pairing $\tilde{\mathrm{A}}^{k}$ in (2) as a global graph-optimization problem. First of all, we consider the complete bipartite graph whose nodes represent the measurement sets $\mathrm{M}^{k-1}$ and $\mathrm{M}^{k}$, and whose edges are represented by $\Omega$. Our goal is to select a subgraph such that the sum of the weights $w_{i j}$ of the edges is maximized subject to the restriction that all vertices in this graph have a degree less or equal to one, so that each target detection from set $\mathrm{M}^{k-1}$ is assigned to at most one target detection on $\mathrm{M}^{k}$ and vice-versa. This set of edges receives the name of matching. It exactly corresponds to the pairing $\mathrm{A}^{k}$ if we appropriately choose the edge weights $w_{i j}$. Despite the fact that we are maximizing a criterion based on graph edges, we are not biasing the solution towards high connectivity (see Fig.2).

This graph-optimization problem corresponds to a known problem in graph theory called maximum weighted bipartite graph matching [10], which can easily be expressed in the context of integer linear programming. In this framework, the objective is to maximize

$$
\sum_{i=1}^{N_{k-1}} \sum_{j=1}^{N_{k}} w_{i j} \chi_{i j}
$$

subject to $\sum_{i=1}^{N_{k-1}} \chi_{i j} \leq 1, \sum_{j=1}^{N_{k}} \chi_{i j} \leq 1$, and $\chi_{i j} \geq 0$.

The solution to the integer linear programming problem would be either $\chi_{i j}=0$ or $\chi_{i j}=1$, where $\chi_{i j}=0$ denotes $(i, j) \notin \mathrm{A}^{k}$ and $\chi_{i j}=1$ denotes $(i, j) \in \mathrm{A}^{k}$. In general, integer linear programming problems are well-known problems considered to be NP-hard. Therefore, there exist many generic algorithms capable of solving them at the expense of high computational costs. Fortunately, the computational complexity can be drastically reduced using graph techniques thanks to the particularities of our very specific case.

In our case, we know beforehand that many pairings between measurements of $\mathrm{M}^{k-1}$ and $\mathrm{M}^{k}$ are highly unlikely. These incompatibilities are usually known when designing the experimental setup (e.g., a cell will not be able to move more than a certain distance, the shape of a target cannot change through time).

In our formulation, the probability of any matching that includes one of these unlikely edges is set to $P\left(\mathrm{~A}^{k}\right)=0$. Therefore, the solution has to be found in a sparse-graph $G$ (see Fig.1b), where the

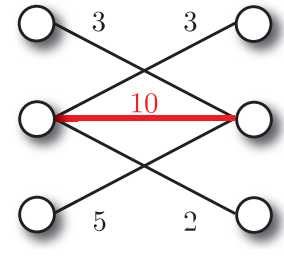

(a)

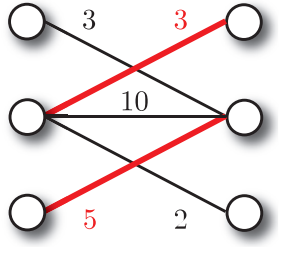

(b)
Fig. 2. A maximum-weight matching (a) is not necessarily a maximum-cardinality matching (b).

edges only connect possible correspondences (i.e., measurements located within a certain region). For all possible matchings within this sparse graph, we can chose a non-zero value for $P\left(\mathrm{~A}^{k}\right)$ such that the solution of (2) corresponds to the solution in (3) with

$$
w_{i j}=-\mu_{S}\left((i, j), \mathrm{S}^{k-1}, \mathrm{~S}^{k}, \mathrm{~A}^{k}\right)-\lambda \mu_{X}\left((i, j), \mathrm{X}^{k-1}, \mathrm{X}^{k}, \mathrm{~A}^{k}\right),
$$

where $\lambda$ is a positive tradeoff parameter between the compatibility measure of the joint movement and the feature compatibility measure. For $\lambda=0$ the solution of the pairing is totally based on target features, and for high values of $\lambda$, the solution is dominated by the joint movement criteria. Note that the compatibility measure of the joint movement acts as a regularization term, that is, modifies the optimal solution of the problem given our prior knowledge of the flow behavior. Therefore, the compatibility measure of the joint movement cannot act independently and should always work together with the feature compatibility measure.

Note that it is necessary to have a pairing $\mathrm{A}^{k}$ to compute the edge weights $w_{i j}$. This can be resolved by the following iterative algorithm:

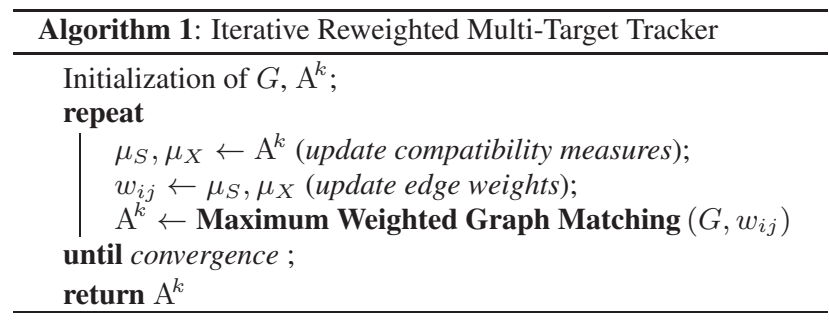

The most costly part of the algorithm corresponds to the execution of the Maximum Weighted Graph Matching function. Some graph algorithms can perform this function in $O\left(N E+N^{2} \log (N)\right)$ steps, where $N=N_{k-1}+N_{k}$ and $E$ is the number of edges (i.e., possible correspondences). A detailed description of the implementation will be presented in the future elsewhere, however, the basic ingredients can be found in [11].

Note the parallelism with the widely used Expectation-Maximization algorithm. The edge-weight update plays the role of the Expectation step, and the graph-optimization step is the counterpart of the Maximization step.

\section{MOTION MODEL}

Blocks of cells often tend to move together in heavy populated environments, so that the cells preserve a certain spatiotemporal continuity in their movement (see Fig. 5). We model this behavior using a displacement model in the compatibility measure of the joint move- 
ment $\mu_{X}$, as follows:

$$
\begin{aligned}
& \mu_{X}\left((i, j), \mathrm{X}^{k-1}, \mathrm{X}^{k}, \mathrm{~A}^{k}\right) \\
& =\frac{1}{\mathcal{N}_{i}^{k-1}} \sum_{\boldsymbol{x}_{l}^{k-1} \in \mathcal{N}_{i}^{k-1}}\|\underbrace{\left(\mathcal{A}\left(\boldsymbol{x}_{l}^{k-1}\right)-\boldsymbol{x}_{l}^{k-1}\right)}_{\text {neighbor } l \text { displacement }}-\underbrace{\left(\boldsymbol{x}_{j}^{k}-\boldsymbol{x}_{i}^{k-1}\right)}_{i \rightarrow j \text { displacement }}\|,
\end{aligned}
$$

where $\mathcal{N}_{i}^{k-1}$ is the non-empty set of neighbors of the detected cell at $\boldsymbol{x}_{i}^{k-1}$, and where $\mathcal{A}$ is a mapping induced by $\mathrm{A}^{k}$ that satisfies $\mathcal{A}\left(\boldsymbol{x}_{i}^{k-1}\right)=\boldsymbol{x}_{j}^{k}$ whenever $(i, j) \in \mathrm{A}^{k}$.

The regularization term $\mu_{X}$ measures the average relative displacement of the neighboring targets given an pairing $\mathrm{A}^{k}$. Its minimization imposes that neighboring target detections go to neighboring target detections (see Fig.3). This term becomes crucial when all targets look alike, which is the case when imaging living cells.

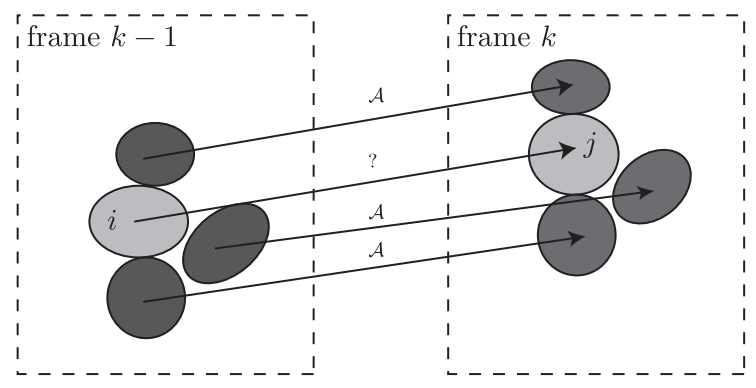

Fig. 3. Schematic representation of the optimal configuration corresponding to the measure $\mu_{X}$. The optimal value is reached when pairing $\boldsymbol{x}_{i}^{k-1}$ with $\boldsymbol{x}_{j}^{k}$, making the movement coherent. As can be seen, the criterion is consistent, even when facing large displacements.

\section{APPLICATION TO TIME-LAPSE MICROSCOPY}

In order to validate the efficacy of the method in tracking individual cells in large crowds, the algorithm was applied to a sequence of images obtained from yeast cell populations.

S. cerevisiae strains (ATCC 201388) were grown within a microfluidic chamber in YPD medium at $30^{\circ} \mathrm{C}$. Monolayer-grown cell crowds were imaged with a Nikon Ti-E microscope (Nikon instruments inc., Melville, U.S.A.), a $60 \times$ objective (plan apo, 1.4 oil), and a $1.5 \times$ scope, resulting in a total $90 \times$ magnification. An iXonEM camera (Andor technology plc., Belfast, U.K.) controlled by a VB6 based software was used to acquire 14-bit images of $1024 \times 1024$ pixels with an exposure time of $50 \mathrm{~ms}$, an analogical gain of 2.4, and an EM gain of 2.

We used watershed-based segmentation [12] to split the image domain in non-overlapping regions (see Fig. 4). This segmentation provides us with several important parameters for the construction of our graph $G$. In particular we identify the centroid of each watershed region as the position $\boldsymbol{x}_{i}^{k-1}$, and the grayscale values within a bounding box covering the watershed region as the features $\boldsymbol{s}_{i}^{k-1}$. The watershed segmentation also gives information about neighboring regions: regions that share dams with other regions are considered neighbors. We use this information to determine the non-empty sets of neighbors in (4). For the watershed region detector to succeed, we need dense areas of targets within crowds. Otherwise, some heuristic methods can be applied to eliminate regions that do not represent cells.

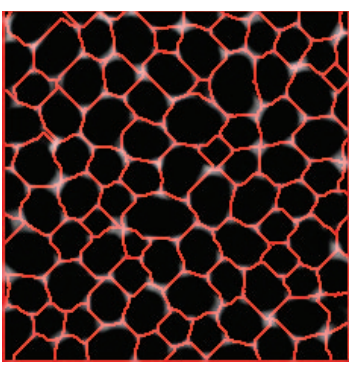

(a)

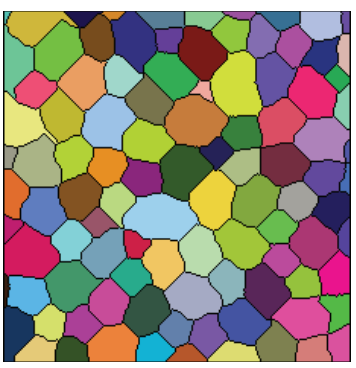

(b)
Fig. 4. Watershed segmentation: (a) original image with overlaid dams (b).

We define the similarity criteria $\mu_{S}$ between two targets as the mean-squared error of the grayscale values between the two corresponding watershed regions. In order to avoid misalignments of the images when computing the MSE, we used the Three-Step Search (TSS) block-matching algorithm [13]. Some typical results are presented in Fig. 5. In the simulations presented in this section we used an initial matching $\mathrm{A}^{k}$ based on features only (i.e. we run the Maximum Weighted Graph Matching function once with $\lambda=0$ ).

To illustrate the adequacy of our motion model, we compared the use of $\mu_{S}$ alone to the joint use of $\mu_{S}$ and $\mu_{X}$ (see Fig. 6c). In this set-up, all targets look very similar, and the $\mu_{S}$ measure is not able to discriminate well between all possible pairings. Introducing $\mu_{X}$ notably improves the solution even after one single iteration.

For the purpose of assessing tracking performances, we manually identified cell trajectories within an area of interest with an average population of 120 yeast cells over a span of 50 frames. We quantified the quality of the pairing process by computing the rate of successful connections made by the algorithm over the total number of connections in each frame. We summarize the results obtained by our method in Table 1.

Table 1. Success rate when tracking yeast cells.

\begin{tabular}{|c|c|c|c|}
\hline$\lambda$ & Max. rate [\%] & Min. rate [\%] & Average rate [\%] \\
\hline \hline 0 & 100.0 & 67.0 & 95.5 \\
\hline 50 & 100.0 & 72.0 & 97.6 \\
\hline 100 & 100.0 & 90.0 & 98.6 \\
\hline
\end{tabular}

The similarity measure $\mu_{S}$ is sufficient for obtaining a correct pairing when cell movements are small (i.e., past and present positions overlap). However, when pressure inside the device exceeds a critical level, clusters of cells displace pushing the neighboring ones. This situation cannot be appropriately handled by using $\mu_{S}$ alone, and tracking is then improved with the introduction of $\mu_{X}$. The appropriate choice of $\lambda$ strongly depends on the behavior of the motion vector field and should be further investigated.

\section{CONCLUSION}

We have presented a new algorithm for multi-cell tracking in crowded areas. It is fast and easy to implement. It runs in real time and is able to deal with thousands of cells. We have specifically designed model-based cost functions that take account of multiple cell interactions. The parameter $\lambda$ of the algorithm needs to be 


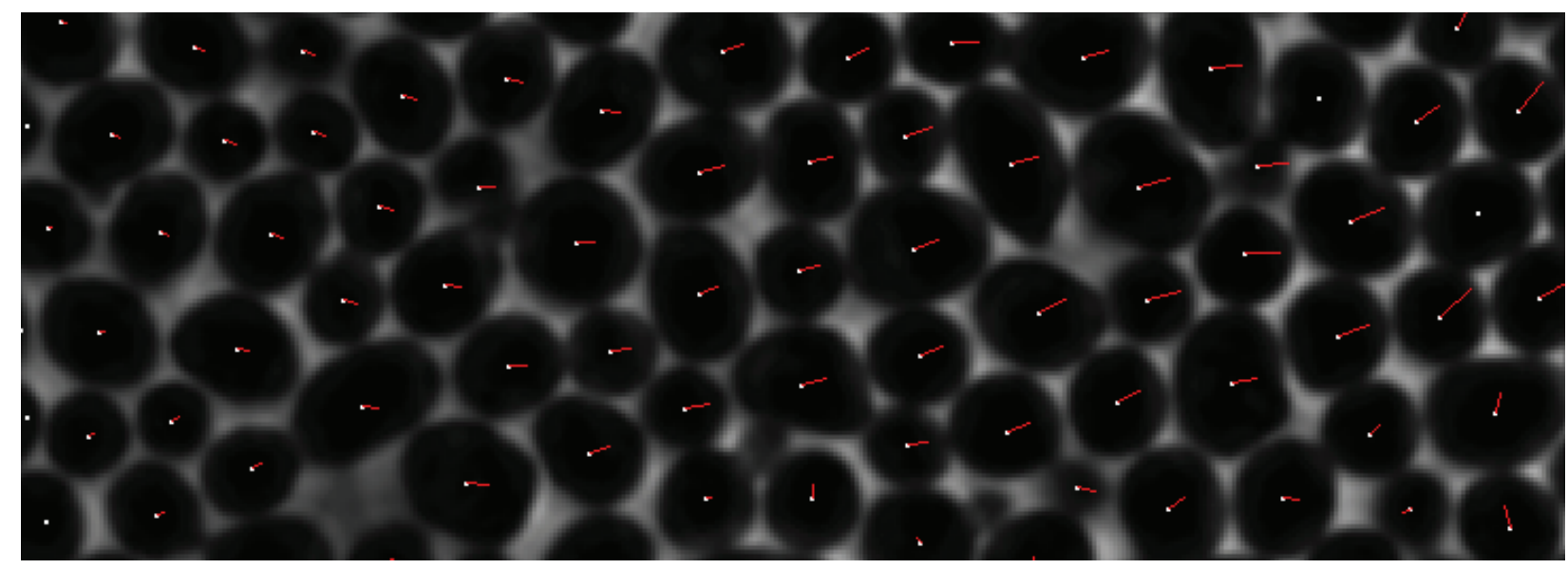

Fig. 5. Flow-like behavior of the constrained movement of a crowd of cells. The red lines point towards the position in the subsequent frame.

chosen so as to strike a balance between the frequently conflicting goals of having flow-like trajectories and enforcing the similarity of individual cells across frames. The question of how to efficiently resolve this tradeoff situation needs further investigation and will be addressed in future work.

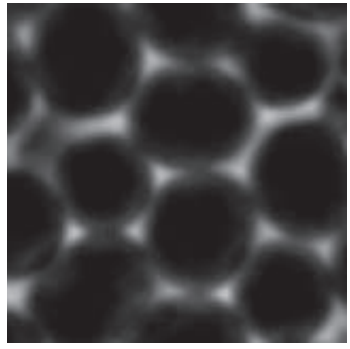

(a)

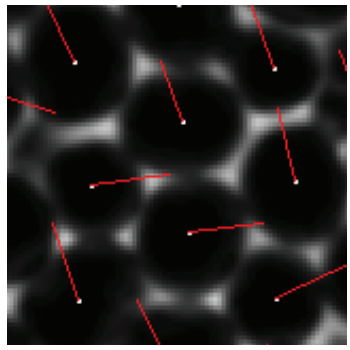

(c)

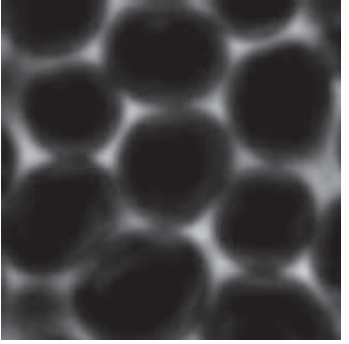

(b)

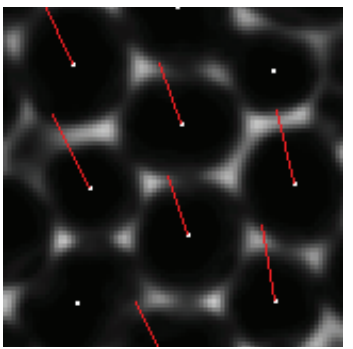

(d)
Fig. 6. Pairing between two consecutive frames. The red lines point towards the location of the corresponding region in the subsequent frame. (a) previous frame $k-1$, (b) current frame $k$, (c) solution based only on the similarity measure $\mu_{S}(\lambda=0)$, (d) solution with a weighted combination of both measures after one iteration using (c) as initial matching $(\lambda=100)$.

\section{REFERENCES}

[1] Q. Wu, F. A. Merchant, and K. R. Castleman, Microscope Image Processing. Elsevier, 2008.
[2] K. Miura, "Tracking movement in cell biology," Adv. Biochem. Engin./Biotechnol., vol. 95, pp. 267-295, 2004.

[3] S. Ali and M. Shah, "Floor fields for tracking in high density crowd scenes," in Proc. ECCV'08: Part II, pp. 1-14, 2008.

[4] K. Li, E. D. Miller, M. Chen, T. Kanade, L. E. Weiss, and P. G. Campbell, "Cell population tracking and lineage construction with spatiotemporal context," Med. Image Anal., vol. 12, pp. 546-566, 2008.

[5] Z. Khan, T. Balch, and F. Dellaert, "MCMC-Based particle filtering for tracking a variable number of interacting targets," IEEE Trans. Pattern Anal. Mach. Intell., vol. 27, pp. 18051918, 2005.

[6] N. Chenouard, I. Bloch, and J.-C. Olivo-Marin, "Feature-aided particle tracking," in IEEE ICIP'08, pp. 1796-1799, Oct. 2008.

[7] N. Chenouard, I. Bloch, and J.-C. Olivo-Marin, "Multiple hypothesis tracking in microscopy images," in IEEE ISBI'09, pp. 1346-1349, July 2009.

[8] P. Vallotton, A. Ponti, C. Waterman-Storer, E. Salmon, and G. Danuser, "Recovery, visualization, and analysis of actin and tubulin polymer flow in live cells: A fluorescent speckle microscopy study," Biophys. J., vol. 85, no. 2, pp. 1289-1306, 2003.

[9] I. Sbalzarini and P. Koumoutsakos, "Feature point tracking and trajectory analysis for video imaging in cell biology," J. Struct. Biol., vol. 151, no. 2, pp. 182-195, 2005.

[10] D. Conte, P. Foggia, C. Sansone, and M. Vento, "Thirty years of graph matching in pattern recognition," Int. J. Pattern Recognit Artif Intell., vol. 18, no. 3, pp. 265-298, 2004.

[11] J. Erickson, "Algorithms course materials." http://compgeom.cs.uiuc.edu/ jeffe/teaching/algorithms/.

[12] P. Soille, Morphological image analysis: principles and applications. Springer-Verlag Berlin Heidelberg 1998, 2003.

[13] T. Koga, K. Iinuma, A. Hirano, Y. Iijima, and T. Ishiguro, "Motion-compensated interframe coding for video processing," in Proc. National Telecommunications Conference, vol. 4, pp. 1-5, 1981. 